\title{
Identification and Detection of Partial Discharge and Electrical Breakdown within a Cavity by using Wavelet Transform under AC Condition
}

\author{
R.S. Pote ${ }^{1}$, V.N. Gohokar ${ }^{2}$, D.G. Wakde ${ }^{3}$ \\ Department of Electrical Engineering, SSGMCOE, Shegaon, India ${ }^{1}$ \\ Department of Electrical Engineering, Shri Shivaji Memorial College of Engineering, Pune, India ${ }^{2}$ \\ P.R.Pote Patil College of Engineering, Amravati, India ${ }^{3}$
}

\begin{abstract}
In high voltage system, the measurement of partial discharge (PD) is used in the assessment of an insulation system. Through modelling the PD process, a better understanding of the phenomenon may be evaluated. In this paper, a model for a cavity within a dielectric material has been developed and tested by using MATLAB environment. The model has been used to study the effect of various applied voltages on the cavity. The measurements were performed for different amplitudes of the applied voltage. The measured results show that PD is strongly influenced by various conditions such as applied voltages, frequencies and the type of the cavity. The cycle to cycle behavior of PD events, discharge phase and magnitude distributions, numbers of PDs per cycle, total charge magnitude per cycle for each set have been obtained and analyzed. The test results from the PD model have been studied and analyzed. It is found that certain model parameters are dependent on the applied voltage, frequencies and cavity conditions. Parameters that clearly affect PD activity can be readily identified.
\end{abstract}

Keywords: Partial Discharge, Corona Discharge, Surface Discharge, Treeing \& Tracing, Void, Cavity, Wavelet Transform, Signal Energy, Breakdown.

\section{INTRODUCTION}

PARTIAL DISCHARGE WITHIN A CAVITY IN A SOLID DIELECTRIC

Partial discharge (PD) is a discharge event that does not bridge the electrodes within an electrical insulation system under high field-stress. When PD happens, discharge starts from one end of the cavity surface, bridging through the gas-filled cavity and reaches the other end of the cavity surface. Thus, PD only bridges the cavity and does not bridge the whole insulation between electrodes. PD normally happens in the presence of a defect within insulation under a high electric field. Examples of defects that may exist in polymeric insulation are voids, cracks, cavities or Partial discharge in a solid dielectric material, usually occurs in gas-filled cavities within the material.

Since the permittivity of the gas is less than the permittivity of the surrounding material, the electric field in the cavity is higher than the material. When the electric field in the cavity is sufficiently high and the breakdown strength of the gas in the cavity is exceeded, PD can occur. During the PD event, the gas changes property from a nonconducting to a conducting medium, resulting in the electric field within the cavity dropping from a higher to a lower value in a very short period of time.

Figure-1 shows a basic diagram of PD within a cavity in a dielectric material which is stressed under a high electric field.

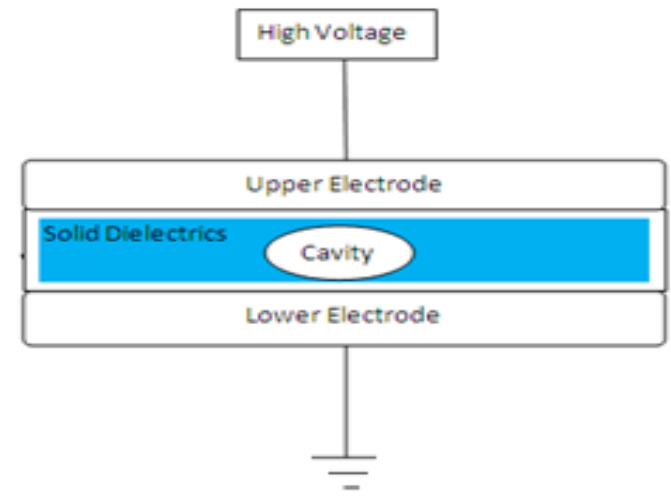

Figure1: Basic diagram of PD within a cavity in a dielectric material

The effect of PD within a cavity in high voltage insulation can be very serious because it can ultimately lead to complete failure of the whole system. Repetition of PD causes progressive chemical deterioration of the material. The chemical transformation of the cavity surface may increase the conductivity of the cavity surface. It may also cause the pressure in the cavity to change due to creation of gaseous by-products, depending on the type of the gas content in the cavity and the material surrounding the cavity. It is theoretically proposed that the cumulative effect of PD in a cavity is the formation of numerous, 


\section{International Journal of Innovative Research in Electrical, Electronics, Instrumentation and Control Engineering \\ ISO 3297:2007 Certified}

Vol. 5, Issue 1, January 2017

branching partially conducting discharge channels in the material, called electrical treeing. Electrical treeing is a significant degradation mechanism that can lead to insulation breakdown and consequently leading to breakdown of the insulation system when the tree channels form a conducting path between the electrodes.

There are several types of discharge other than partial discharge, including surface discharge and corona discharge. Surface discharge is a discharge event that occurs on an insulating surface where the tangential field across the surface is high. This discharge can bridge the potential difference between the high voltage source and the ground electrode through cracks or contaminated paths on the insulation surface. Examples of surface discharge in the field or on the insulation surface of a high voltage cable or at the end-windings of stator windings of large generators. Corona discharge is discharged in gas due to a locally enhanced field from a sharp point of an electrode which ionizes the surrounding gas molecules.
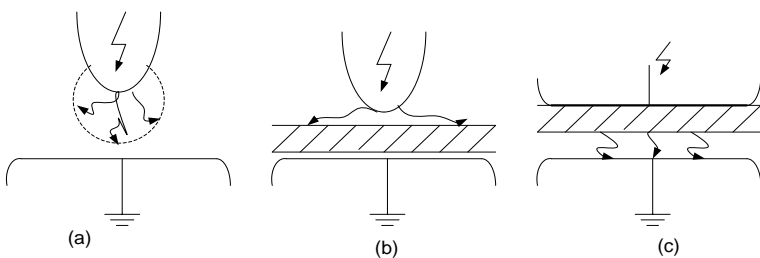

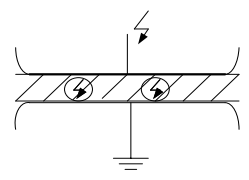

(d)

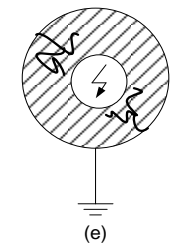

Figure2: Various discharges a) Corona discharge $b$ ) Surface discharge d) Partial discharge

FACTORS AFFECTING PARTIAL DISCHARGE (PD) PD activity in a solid dielectric material depends upon applying voltage, dielectric constant of material and size of the void. These are considered as the factors affecting Partial Discharge in solid dielectrics

\section{Applied voltage}

When the applied voltage is increased, the electric field is enhanced and the electron generation rate is increased. As a result more PDs will occur. High voltage ranging from 4 $\mathrm{KV}$ to $20 \mathrm{KV}$ is applied to the simulation model to observe PD activity due to the presence of void, which is done using Matlab/Simulink.

\section{Different sample materials}

Depending upon different materials used in the insulation model the apparent charge varies. Parameters in Table.1 are simulated using MATLABto observe the variation of Partial Discharge with different materials used in the sample model. Each material has different dielectric constants.
TABLE-I: Different Sampling Materials

\begin{tabular}{|l|l|}
\hline Material & $\begin{array}{l}\text { Dielectric } \\
\text { constant } \mathbf{~ r ~}\end{array}$ \\
\hline Air & 1 \\
\hline Alumina porcelain & 10 \\
\hline Bakelite & $3.5-5.0$ \\
\hline $\begin{array}{l}\text { Cross-Linked Polyethylene } \\
\text { (XLPE) }\end{array}$ & 2.4 \\
\hline Epoxy Resin & 3.6 \\
\hline Glass & $3.7-10$ \\
\hline Hard paper (Laminated) & 4.5 \\
\hline Marble & 8 \\
\hline Mica & $2.5-7.0$ \\
\hline Oil paper & 4 \\
\hline Paper & $2-3$ \\
\hline Paper Impregnated & 5 \\
\hline Polypropylene resin & 2 \\
\hline Polyvinyl Chloride (PVC) & 3.4 \\
\hline Porcelain & $5-7$ \\
\hline Press board & 4 \\
\hline Rubber & 3 \\
\hline Transformer oil (Mineral) & 2.2 \\
\hline Vegetable oil & 2.5 \\
\hline Vulcanized Rubber & 7 \\
\hline Wood (Dry) & $2-6$ \\
\hline
\end{tabular}

3. Different void size

In the insulation of power equipment, voids are one of the main factors which cause PD and its variation in size has great effect on the characteristics of PD. The lifetime of insulation, very much depended on the size of the void. Smaller size of void takes longer time to harm the insulation. Using Matlab simulated the behavior of PD in insulation system with different void size is studied.

\section{PARTIAL DISCHARGE CAPACITANCE MODELS}

MODELLINGTHREE

A partial Discharge (PD) model using a three-capacitor circuit model or ' $a-b-c$ ' model representing an isolated cavity within a dielectric material has been developed. Discharge is represented by an instantaneous change in the charging of a capacitance in the test object. A similar model has been used to study PD behavior. The statistical behavior of this three-capacitance circuit is very complex, even though the circuit is simple and deterministic.

However, this model is not realistic in describing cavity properties because in a real cavity, there is surface charge accumulation on the cavity surface after a discharge occurs and the cavity surface is not an equipotential surface. There is an improved 'a-b-c' model which has considered charging accumulation on the cavity surface after a discharge. The discharge is simulated as a time and voltage dependent resistance, which represents the discharge event as a change in the cavity from being insulating to conducting. 
The figure 3 shows the typical three-capacitance equivalent circuit or 'abc' model of a cavity within a dielectric material. $\mathrm{C}_{\mathrm{a}}$, and $\mathrm{C}_{\mathrm{a}}$ " represent the capacitance in the material which is cavity-free, $\mathrm{C}_{\mathrm{b}}$, and $\mathrm{C}_{\mathrm{b}}$ " represent the capacitance in the material in series with the cavity, $\mathrm{C}_{c}$ represents the cavity capacitance.

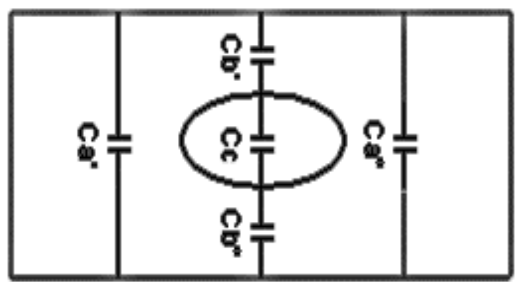

Figure3: Typical three-capacitance equivalent circuit or 'abc' model

The simplified equivalent circuit can be derived from the geometry, as shown in Figure 4 , where $C_{a}$ is the equivalent parallel capacitance of $\mathrm{C}_{\mathrm{a}}$, and $\mathrm{C}_{\mathrm{a}}, \mathrm{C}_{\mathrm{b}}$ is the equivalent series capacitance of $\mathrm{C}_{b}$, and $\mathrm{C}_{b}$, and $\mathrm{V}_{\mathrm{c}}$ is the voltage across the cavity. Discharge is assumed to occur when the voltage across the cavity capacitance $V_{c}$ is higher than the inception voltage, $V_{\text {inc }}$ and stops when it is less than the extinction voltage, $\mathrm{V}_{\text {ext }}$. When a discharge occurs, $\mathrm{C}_{\mathrm{c}}$ is short circuited, causing a fast transient current to flow in the circuit due to the voltage difference between the voltage source and across $C_{b}$. A fast transient voltage signal is created due to sudden voltage drop due to the impedance of the external circuit.

Although this model is simple, it can represent the transient related to a discharge.
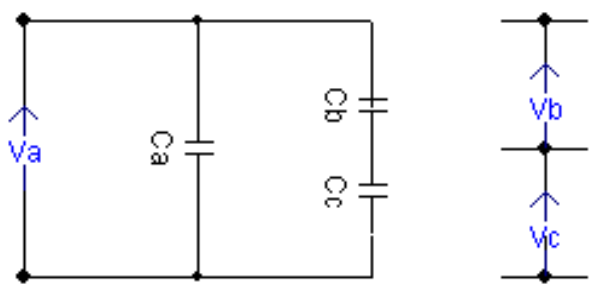

Figure4: The simplified equivalent circuit

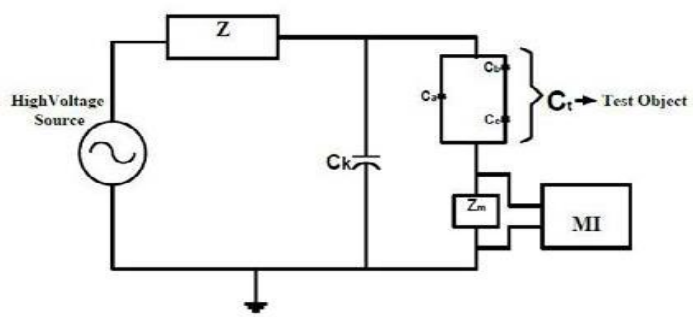

Figure5: The Experimental/Simulation arrangement of the simplified equivalent circuit

The three capacitance equivalent circuit diagram representing PD in a cavity. The PD current pulse and apparent charge magnitude as a function of time, which results from a voltage across the cavity and the current flowing through the cavity due to the partial discharge

\section{PD CHARGE MAGNITUDE}

In this model, since the discharge process is modelled. The charge magnitude can be calculated numerically. The real and apparent charge magnitudes, $\mathrm{q}_{\mathrm{pd}}$ are calculated by time integration of current, $\mathrm{I}(\mathrm{t})$ owing through the cavity and through the ground electrode during the- PD time interval, Where $\mathrm{q}_{\mathrm{pd}}=\int_{\mathrm{t}}^{\mathrm{t}+\mathrm{dt}} \mathrm{I}(\mathrm{t}) \mathrm{dt}$

The current I, through the ground electrode is calculated by integration of current Density, $\mathrm{J}$ over the ground electrode surface area, where $\mathbf{J}$ depends on the electric field distribution. Since the electric field distribution on the ground electrode is not uniform due to the presence of the cavity, the field distribution in the whole cavity and the material is calculated using the MATLABmethod to determine the PD apparent charge magnitude. Therefore, the advantage of the use of MATLABover classical lumped parameter modelling is that it facilitates dynamic calculation of both real and apparent charges.

\section{BREAKDOWN DUE TO INTERNAL DISCHARGES}

Solid insulating materials contain voids or cavities within the medium or at the boundaries between the dielectric and the electrodes. These cavities are generally filled with a medium of lower dielectric strength, and the dielectric constant of the medium in the voids is lower than that of the insulation. The electric field strength in the voids is higher than that across the dielectric. Therefore, even under normal working voltages the field in the voids may exceed their breakdown value, and breakdown may occur. Let us consider a dielectric between two conductors as shown in Figure 4, where, $\mathrm{C}_{\mathrm{c}}$ : capacitance of the void or cavity. $\mathrm{C}_{\mathrm{b}}$ : capacitance of the dielectric which is a series with the void and $\mathrm{C}_{\mathrm{a}}$ : capacitance of the rest of the dielectric.

The voltage across the void, $\mathrm{V}_{\mathrm{C}}$ is given by,

$$
\mathrm{Vc}=\frac{\mathrm{V} * \mathrm{~d} 1}{\mathrm{~d} 1+\left(\frac{\epsilon 0}{\epsilon 1}\right) \mathrm{d} 2}
$$

Where, $\mathrm{d} 1$ and $\mathrm{d} 2$ are the thickness of the void and the dielectric respectively, having permittivity $\mathrm{E} 0$ and $\mathrm{Cr}$. Usually $\mathrm{d} 1<<\mathrm{d} 2$, and we assume the cavity is filled with a gas, then;

$$
\mathrm{V} 1=\mathrm{V} * \operatorname{Cr}\left(\frac{\mathrm{d} 1}{\mathrm{~d} 2}\right)
$$

Where $\mathrm{Cr}=$ the relative permittivity of dielectric.

When a voltage $V_{a}$ is applied, $V_{a}$ reaches the breakdown strength of the medium in the cavity $\mathrm{V}_{\mathrm{c}}$ and breakdown occurs. $V_{c}$ is called the "discharge inception voltage". When the applied voltage is AC, breakdown occurs on both the half cycles and the number of discharges will depend on the applied voltage. The voltage and the discharge current waveforms are shown in Figure. These internal discharges (also called partial discharges, PD) have the same effect as treeing on the insulation. 


\section{WAVELET TRANSFORM}

\section{A. ERROR SIGNAL GENERATION}

The error signal generation block extracts the superimposed distortions on the measured voltages. The error signal is obtained by subtracting the fundamental component of the input signal.

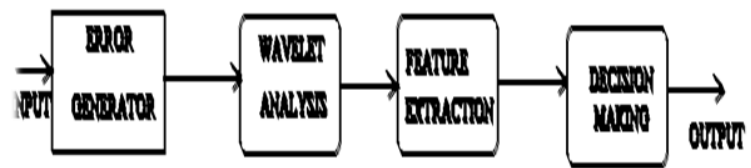

Figure 6: Proposed Disturbance Detection Scheme

\section{B. WAVELET TRANSFORM ANALYSIS}

The wavelet analysis block transforms the error signal into different time-frequency scales. The wavelet transform provides information about the frequency content of a signal similar to the Fourier Transform (FT). However, contrary to the FT, wavelet transform is able to focus on short time Intervals for high-frequency components and long intervals for low-frequency components, thus making it a well suited tool for analyzing high-frequency transients in the presence of low-frequency components. The Wavelet transform is inherently more appropriate for non-stationary and non-periodic wideband signals.

The wavelet transform of a continuous signal is:

$$
F(a, b)=\frac{1}{\sqrt{a}} \int_{-\infty}^{\infty} f(t) \Psi\left(\frac{t-b}{a}\right) d t
$$

Where

$\Psi$ is the wavelet basis function (mother wavelet), $a$ is the time scaling factor and

$\mathrm{b}$ is the time shifting factor.

The values of wavelet coefficients $F(a, b)$ represent the projection of $f(t)$ along $\Psi\left(\frac{t-b}{a}\right)$. Assume the center and width of function $\Psi(\mathrm{t})$ is zero and $\Delta_{\mathrm{t}}$ in time domain, and zero and $\Delta_{\mathrm{w}}$ in the frequency domain. Then, the function $\Psi\left(\frac{t-a}{b}\right)$ is centered at band has width of $a \Delta_{t}$ in time domain and (1/a) $\Delta_{\mathrm{w}}$ in the frequency domain. To avoid generating redundant information, the base functions are generated discretely by selecting $a=a_{0}{ }^{m}$ and $b=$ $\mathrm{nb}_{0} * \mathrm{a}_{0}{ }^{\mathrm{m}}$

Where, $\mathrm{a}_{0}$ and $\mathrm{b}_{0}$ are fixed constants with $\mathrm{a}_{0}>1$ and $\mathrm{b}_{0}>0$, $\mathrm{m}, \mathrm{n}, \varepsilon, \mathbb{Z}$, and $\mathbb{Z}$ is the set of integers. Setting $\mathrm{a}_{0}$ and $\mathrm{b}_{0}$ to 2 and 1 respectively results in an orthonormal basis of $\mathrm{L}^{2}$ (IR) which is called dyadic- orthonormal wavelet transform. With this ortho-normal basis, an algorithm of decomposing a signal into different time-frequency scales can be used which is called Multiresolution Signal Decomposition (MSD).

\section{B1. WAVELET IMPLEMENTATION}

The filter bank configuration of Fig.2 is used to implement the wavelet transform based on the MSD technique. At the first stage, the error signal is decomposed into $c_{1}$ and $s_{1}$ which represent smoothed and detailed versions of the main signal respectively. In the next step, the smoothed signal is decomposed into $c_{2}$ and $s_{2}$. The process of decomposing can be continued as many stages as required by the application. The relationship between filters and scaling/wavelet functions $(\Phi(\mathrm{t}) / \Psi(\mathrm{t}))$ can be written as

$$
\begin{aligned}
& \phi(\mathrm{t})=\sqrt{2} \sum_{\mathrm{n}} \mathrm{h}[\mathrm{n}] \phi(2 \mathrm{t}-\mathrm{n}) \\
& \Psi(\mathrm{t})=\sqrt{2} \sum_{\mathrm{n}} \mathrm{g}[\mathrm{n}] \phi(2 \mathrm{t}-\mathrm{n})
\end{aligned}
$$

In general output $c_{\mathrm{m}}$ and scale $\mathrm{s}_{\mathrm{m}}$ are expressed as:

$\mathrm{c}_{\mathrm{m}}[\mathrm{n}]=\mathrm{c}_{\mathrm{m}-1}[\mathrm{n}] * \mathrm{~h}\left[\mathrm{n} / 2^{\mathrm{m}-1}\right]$

$\mathrm{s}_{\mathrm{m}}[\mathrm{n}]=\mathrm{c}_{\mathrm{m}-1}[\mathrm{n}] * \mathrm{~g}\left[\mathrm{n} / 2^{\mathrm{m}-1}\right]$

Where:

$$
\begin{aligned}
& \mathrm{c}_{0}[\mathrm{n}] \stackrel{\triangleq}{=} \mathrm{e}[\mathrm{n}] \\
& h\left(\frac{n}{2}\right)=\left\{\begin{array}{cc}
h[k] & { }_{n} n=2 k \\
0 & \text { else }
\end{array}\right.
\end{aligned}
$$

And $*$ is the convolution sign after transforming the error signal into different scales $\left(2^{j}\right)$, the next step is to define features of the signal.

\section{B2. PROPERTIES OF MOTHER WAVELET}

Wavelets are families of functions generated from one single function, called as an analyzing wavelet or mother wavelet.

The mother wavelet must have the following properties:

a) It must be oscillatory

b) It must quickly decay to zero

c) It must have a zero average

d) It must be band- pass

e) It must be integrated to zero

\section{B3. ADVANTAGE OF WAVELET TRANSFORM} OVER FOURIER TRANSFORM

- Wavelet provide greater resolution in time for high frequency components of a signal and greater resolution in frequency for low frequency components of a signal.

- Wavelet performs better with non-periodic signals that signals that contains short duration impulse components as is typical in power systems transients.

- Wavelet has a window that automatically adjusts to give appropriate resolutions.

- Different from fast Fourier Transform, the wavelet transform is approach is more efficient in monitoring various disturbances as time varies.

- Wavelet uses a short window at high frequencies and long window at low frequencies.

- The iterative wavelet method for system converges rapidly for cases of interest in power engineering. 
Vol. 5, Issue 1, January 2017

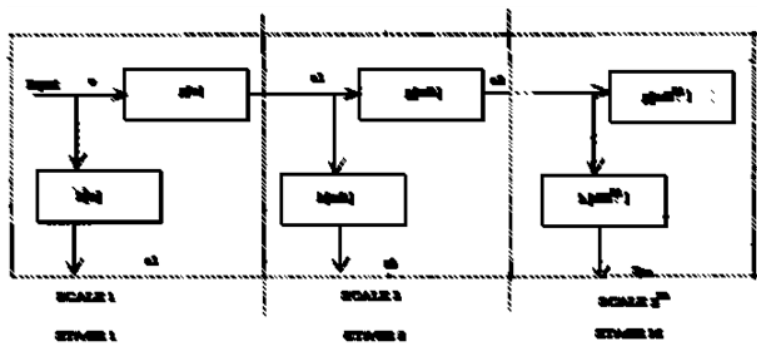

Figure 7:- Realization of wavelet transforms using a filter bank.

\section{FEATURE EXTRACTION}

The purpose of the feature extraction block is to identify Specific signatures of the disturbances in the system. For example, a short circuit and a capacitor-switching incident result in disturbed voltages with different features. The wavelet transform breaks down the error signal into different time-frequency scales. Each scale represents the error signal in the corresponding band. The energy content of the scale signals relative to the error signal changes depending upon the type of disturbance. Therefore, the relative amplitudes of the scale signals with respect to the error signal are selected as the discriminating features.

\section{DECISION MAKING}

A function of the decision making block is to discriminate type of disturbances (L-G fault and Capacitor switching) as precisely as possible.

The characteristic of each disturbance, example a faulty, Capacitor switching, depends on several factors, for example (1) type of event, e.g. single-phase-to-ground or phase-to-phase fault,(2) location of the event, (3) time instant of event and (4) network configuration.

In the decision making block, a probability function is defined for the features and the decisions is made using the maximum linked (ML) criteria. This method is used here to discriminate various types of disturbances in a power system.

\section{EXPERIMENTAL SET- UP ARRANGEMENT}

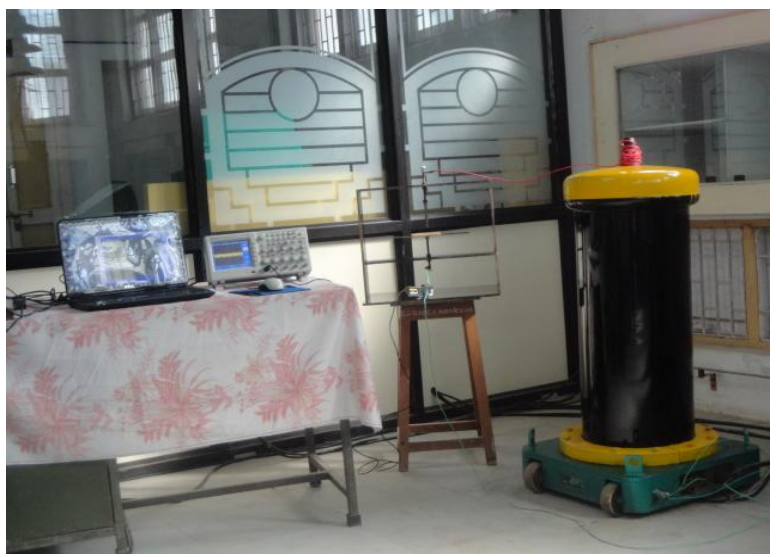

Figure8: Arrangement of Experimental setup

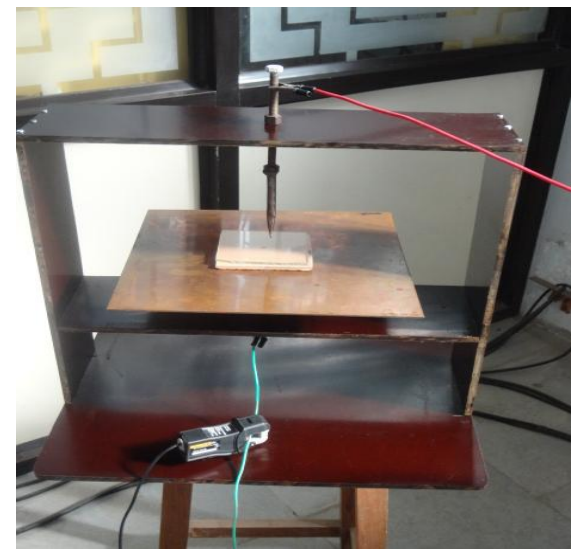

Figure 9: Sample under testing

RESULTS \& DICUSSION

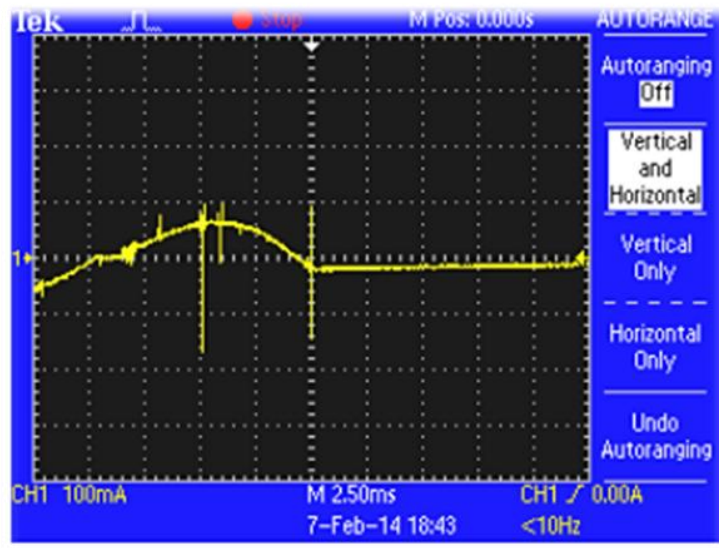

TDS 2014B - 3:47:55 PM 07/02/2014

Figure10: Signal received from laboratory sample testing

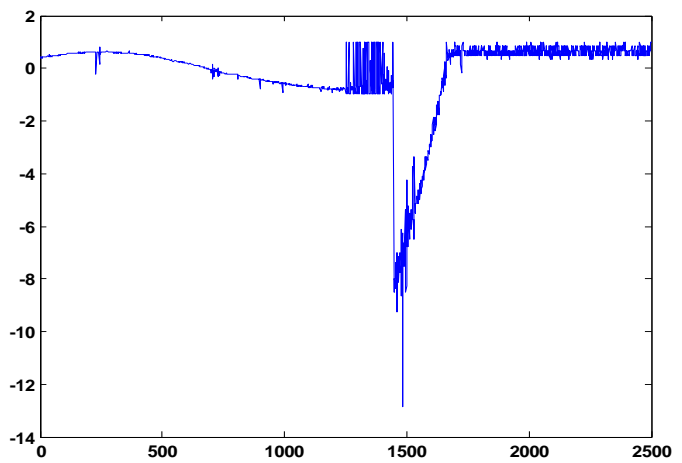

Figure 11: Original partial discharge Signal

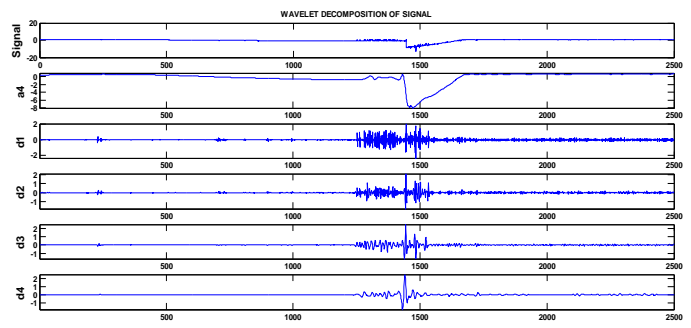

Figure 12: Db4, Decomposition level-4 
IJIREEICE

International Journal of Innovative Research in Electrical, Electronics, Instrumentation and Control Engineering

ISO 3297:2007 Certified

Vol. 5, Issue 1, January 2017

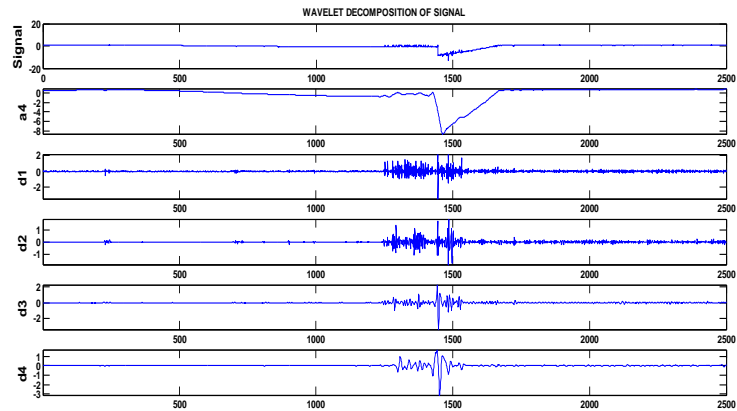

Figure 13: Using sym4

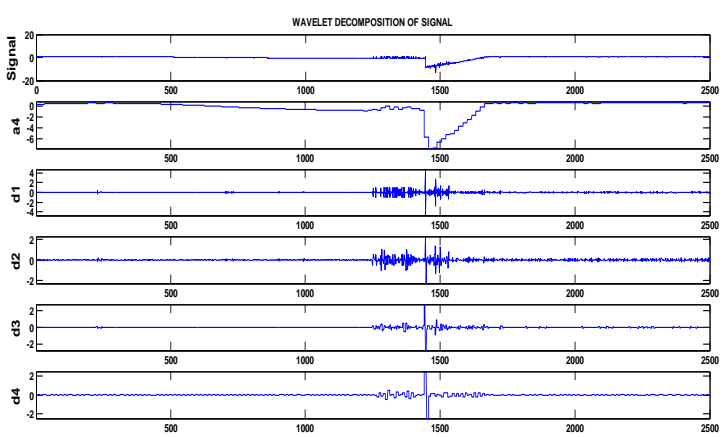

Figure 14: Using Haar
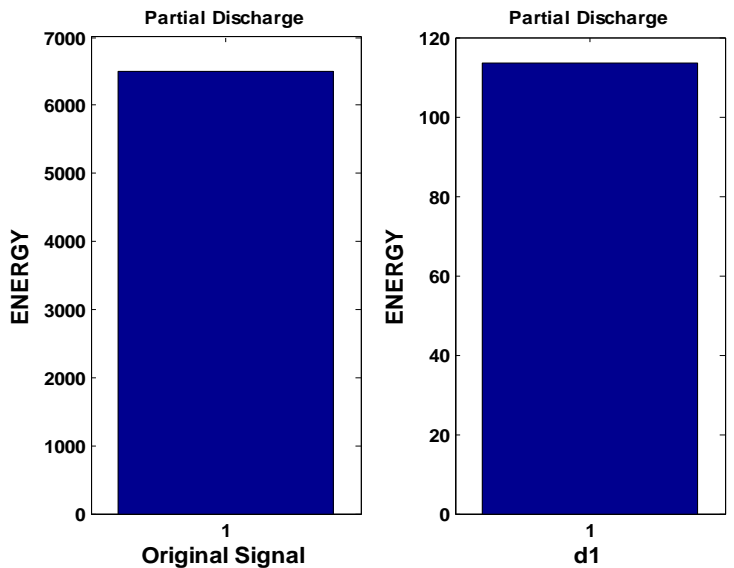

Figure 15: Signal Energy using db4
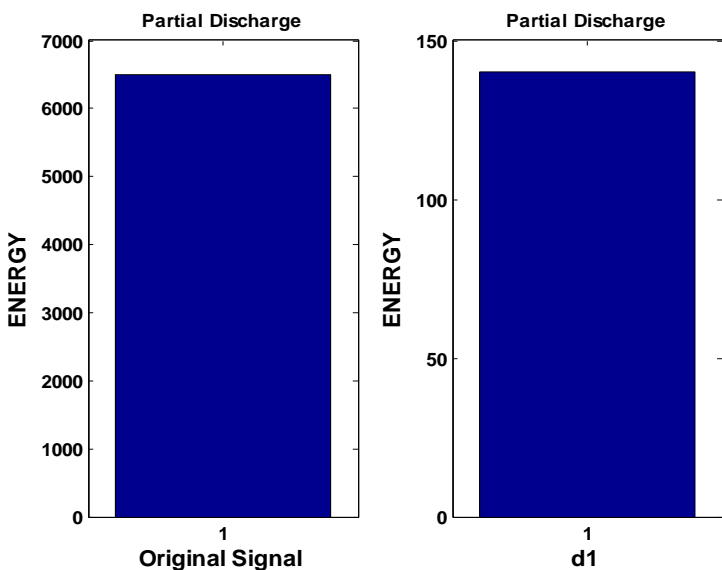

Figure 16: Signal Energy using Sym4
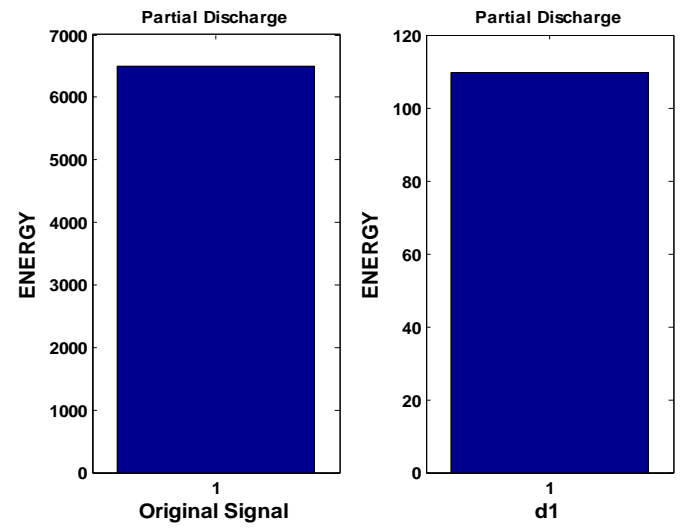

Figure 17: Signal Energy using Haar

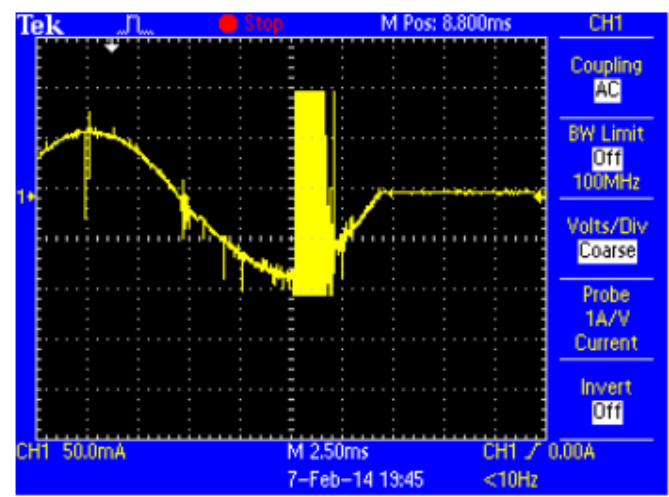

TDS 2014B - 4:50:42 PM 07/02/2014

Figure 18: Signal Captured using DSO

The simulation results are formulated for the partial discharge characteristics detection for both solid and liquid insulating materials from the experimental setup using the partial discharge detector \& it is observed that when external high voltage applied through the separate source \& amplitude of the partial discharges are higher \& detected earlier in the solid insulator. The breakdown occurs earlier in both the configuration in the solid insulator than the liquid insulating oil. Similarly from the simulation results it is also observed that the amplitude of the partial discharges is more in the solid insulator and occurs at the low range of the applied high voltages.

Thus, it is suggested that the partial discharges are more in the solid material than the liquid insulating material due to the presence of the air void and others impurities.

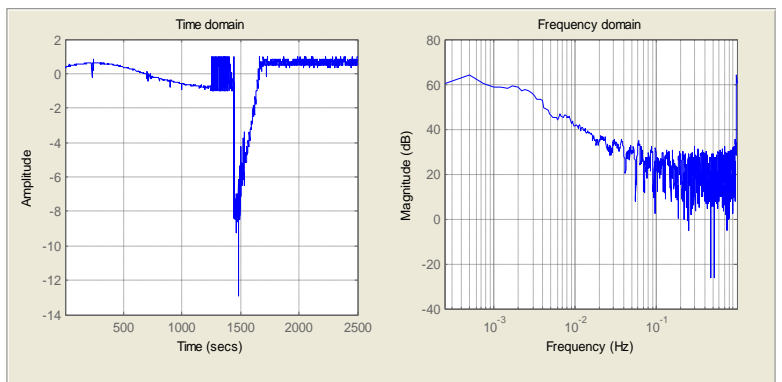

Figure 19: Frequency and time domain of captured signal 


\section{International Journal of Innovative Research in Electrical, Electronics, Instrumentation and Control Engineering ISO 3297:2007 Certified}

Vol. 5, Issue 1, January 2017

Thus, on the basis of partial discharge characteristics oil is a good insulating material than the solid material and can be used in the high voltage equipment's for a specific application that is for low partial discharge requirements.

\section{CONCLUSION}

Partial discharge is the main problem in high voltage power equipment system. Therefore, detection and measurement of partial discharge are necessary to keep the equipments in healthy condition during their operation. In this work simulation study had been carried out for partial discharge measurement and detection using the MATLAB. From the simulation studies, it is observed that in each case the partial discharge detects earlier at the low values of applied high voltages and insulation breakdown occurs at the lower range of high voltage in case of solid insulating material than the oil. So it is concluded that the amplitude of partial discharges is more on the solid insulating materials when the applied voltage is increased because of the presence of air voids and other impurities. MATLABbased model has been developed from the equivalent electrical model of an insulators to observe the characteristics of partial discharge activity inside the solid and liquid insulator at different applied voltage which arranges from $0-20 \mathrm{KV}$ at a constant power frequency $(\mathrm{f}=50 \mathrm{~Hz})$. It is found that with the increase in applied voltage to the void present inside the insulation, partial discharge increases in solid dielectric material.

This work can also be extended in the future for different high voltage power equipment model for detecting the Partial Discharge activity. Further the collected Partial Discharge pulse can be processed with the help Wavelet Transform for time, frequency analysis for better study of PD measurement and detection

\section{REFERENCES}

[1] Illias, H. A., Chen, G. and Lewin, P. L, "Modelling of partial discharge activity in spherical cavities within a dielectric material," IEEE Electrical Insulation Magazine, vol. 27(1), p. 38-45, 2011.

[2] Illias, H. A., Chen, G. and Lewin, P. L, "Partial Discharge Behaviour of a Spherical Cavity within a Solid Dielectric Material as a Function of Applied Voltage and Frequency' IEEE Transactions on Dielectrics and Electrical Insulation, vol.18(2), p. 432-443, 2011.

[3] M. G. Danikas, I. W. McAllister, G. C. Crichton, and A. Pedersen, "Discussion: partial discharges in ellipsoidal and spheroidal voids," IEEE Transactions on Electrical Insulation, vol. 26, no. 3, pp. 537$539,1991$.

[4] A. Pedersen, G. C. Crichton, and I. W. McAllister, "The theory and measurement of partial discharge transients," IEEE Transactions on Electrical Insulation, vol. 26, no. 3, pp. 487-497, 1991.

[5] L. Niemeyer, "A generalized approach to partial discharge modelling," IEEE Transactions on Dielectrics and Electrical Insulation, vol. 2, no. 4, pp. 510-528, 1995.

[6] F. Guteisch and L. Niemeyer, "Measurement and simulation of PD in epoxy voids," IEEE Transactions on Dielectrics and Electrical Insulation, vol. 2, no. 5,pp. 729-743, 1995.

[7] C. Forssen and H. Edin, "Partial discharges in a cavity at variable applied frequency part 2: Measurements and modelling," IEEE Transactions on Dielectrics and Electrical Insulation, vol. 15, no. 6, pp. 1610-1616, 2008.
[8] T. Okamoto and T. Tanaka, "Novel partial discharge measurement computer-aided measurement systems," IEEE Transactions on Electrical Insulation, vol. 21, no. 6,pp. 1015-1019, 1986.

[8] C. S. Kim, T. Kondo, and T. Mizutani, "Change in PD pattern with aging," IEEE Transactions on Dielectrics and Electrical Insulation, vol. 11 , no. 1, pp.13-18, 2004.

[9] R. Bodega, P. H. F. Morshuis, M. Lazzaroni and F. J. Wester, "PD recurrence in cavities at different energizing methods," IEEE Transactions on Instrumentation and Measurement, vol. 53, no. 2, pp. 251-258, 2004

[9] E. Kuffel, W. Zaengl, and J. Kuffel, High Voltage Engineering: Fundamentals, 2nd ed. Butterworth-Heinemann, 2000.

[10] S. A. Boggs, "Partial discharge cavity-induced PD in solid dielectrics," IEEE Electrical Insulation Magazine, vol. 6, no. 6, pp. $11-16,19-20,1990$.

[11] S. A. Boggs, "Partial discharge: overview and signal generation," IEEE Electrical Insulation Magazine, vol. 6, no. 4, pp. 33-39, 1990

[12] Z. Achillides, G. Georghiou, and E. Kyriakides, "Partial discharges and associated transients: the induced charge concept versus capacitive modelling," IEEE Transactions on Dielectrics and Electrical Insulation, vol. 15, no. 6, pp. 1507-1516,2008.

[13] W. Hauschild, A. Cavallini, and G. C. Montanari "Effect of supply voltage frequency on testing of insulation system," IEEE Transactions on Dielectrics and Electrical Insulation, vol. 13, no. 5, pp. 1189-1191, 2006.

[14] E. Lindell, T. Bengtsson, J. Blennow, and S. M. Gubanski, "Measurement of partial discharges at rapidly changing voltages," IEEE Transactions on Dielectrics and Electrical Insulation, vol. 15, no. 3, pp. 823-831, 2008.

[15] B. Florkowska, M. Florkowski, J. Furgal, and P. Zydron, "Influence of different voltage waveforms on PD formation in HV insulation systems," Electrical Insulation Conference, pp. 51-54, 2009

[16] W. Kai, T. Okamoto, and Y. Suzuoki, "Effects of discharge area and surface conductivity on partial discharge behaviour in voids under square voltages," IEEE Transactions on Dielectrics and Electrical Insulation, vol. 14, no. 2, pp. 461, 2007

[17] P. Das and S. Chakravorti, "Studies on partial discharge simulation based on a stochastic model considering the variation of discharge area and temperature of the void surface," International Journal for Computational Methods in Engineering Science and Mechanics, vol. 10 , no. 5, 2009.

[18] H. A. Illias, G. Chen, and P. L. Lewin, "Partial discharge behaviour within a spherical cavity in a solid dielectric material as a function of frequency and amplitude of the applied voltage," IEEE Transactions on Dielectric and Electrical Insulation, vol. 18, no. 2, pp. 432-443, 2011.

[19] H. A. Illias, G. Chen, and P. Lewin, "Modelling of partial discharge activity in spherical cavities within a dielectric material," IEEE Electrical Insulation Magazine, vol. 27, no. 1, pp. 38-45, 2011.

[20] A. A. Ganjovi, N. Gupta, and G. R. G. Raju, "A kinetic model of a PD pulse within voids of sub-millimetre dimensions," IEEE Transactions on Dielectrics and Electrical Insulation, vol. 16, no. 6, pp. 1743-1754, 2009.

[21] H. A. Illias, G. Chen, and P. L. Lewin, "Modelling of partial discharges from a spherical cavity within a dielectric material under variable frequency electric fields," Conference on Electrical Insulation and Dielectric Phenomena, pp. 447-450, 2008.

[22] H. A. Illias, G. Chen, and P. L. Lewin, "Partial discharge modelling in a spherical cavity within a dielectric insulation material as a function of frequency," IEEE Electrical Insulation Conference, pp. $55-59,2009$.

[23] C. Forssen and H. Edin, "Partial discharges in a cavity at variable applied frequency part 1: Measurements," IEEE Transactions on Dielectrics and Electrical Insulation, vol. 15, no. 6, pp. 1601-1609, 2008.

[24] R. Miller and I. A. Black, "Partial discharge measurements over the frequency range $0.1 \mathrm{~Hz}$ to $50 \mathrm{~Hz}$," IEEE Transactions on Electrical Insulation, vol. EI-12, no. 3, pp. 224-233, 1977.

[25] T. Do, O. Lesaint, and J. L. Auge, "Streamers and partial discharge mechanisms in silicone gel under impulse and ac voltages," IEEE Transactions on Dielectrics and Electrical Insulation, vol. 15, no. 6, pp. 1526-1534, 2008. 


\section{IJIREEICE \\ International Journal of Innovative Research in Electrical, Electronics, Instrumentation and Control Engineering \\ ISO 3297:2007 Certified \\ Vol. 5, Issue 1, January 2017}

\section{BIOGRAPHIES}

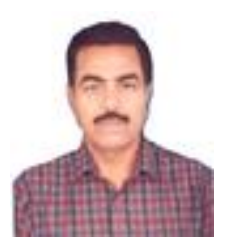

Ravindra Shankarrao Pote received his B.E. (EPS) and M.E. (EPS) degrees from S.G.B. Amravati University, Amravati, India in Electrical Power System Engineering and pursuing his Ph.D. from the same university in Dielectrics and Electrical Insulation. $\mathrm{He}$ is currently working with S.S.G.M. College of Engineering Shegaon, India, as an Associate Professor in Electrical Engineering Department. $\mathrm{He}$ is IEEE, ISCEE (ROORKEE) and ISTE (New Delhi) members. His research interests include Electrical Insulation\& Dielectrics, digital protection, FACTS and power quality, power system planning, operation and control, Energy Conservation \& Management.

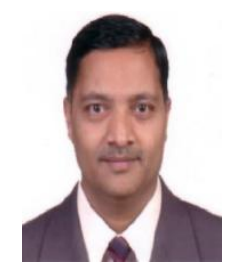

Dr. Vijay Nanaji Gohokar received his B.E. from S.G.B. Amravati University, Amravati, India in 1987, and M.Tech (IPS) from Visvesvaraya National Institute of Technology, Nagpur, India in 1990. Ph.D. Degree in Electrical Engineering from S.G.B. Amravati University, Amravati, India. He is IEEE, ISTE members. His research interests include Automation and Distribution, power system planning, operation and control, stability of the power system, digital Power system protection.

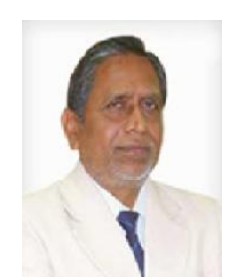

Dr. Dinkar Govindrao Wakde received his M.Sc. (1977) and Ph.D. (1981) from Nagpur University. He isa life member of ISTE, IETE, CSI, IEEE-USA, TERI, ISCE-Ahmedabad. He has 27 years of Teaching Experience. His area of research is signal\& system, material science and electromagnetic. 